\title{
Sodium valproate in psychiatric practice: time for a change in perception
}

\author{
David Cunningham Owens
}

\section{Summary}

Sodium valproate and related preparations have recently undergone regulatory review following concern about effects on the unborn child and doctors' failure to communicate risk. The issues are wider. Valproate is overused in psychiatry based on the false perception that 'ease' of use equates to better safety than alternatives. Valproic acid can disrupt fundamental physiological processes, the consequences of which are poorly understood and little discussed in the psychiatric literature. valproate may be useful in a small number of patients with bipolar disorder but current prescribing patterns are unjustified. Perception needs to change.

\section{Declaration of interest}

D.C.O. is psychiatric commissioner on the Commission on Human Medicines and a member of the European Medicines Agency's Scientific Advisory Group on Psychiatry. He chaired the European Medicines Agency's review of the psychiatric use of valproate in pregnancy and women of childbearing potential.

\section{Keywords}

Sodium valproate; teratogenicity; risk benefit; bipolar affective disorders; perinatal psychiatry.

\section{Copyright and usage}

(C) The Royal College of Psychiatrists 2019
David Cunningham Owens (pictured) is Professor of Clinical Psychiatry at the University of Edinburgh and an Honorary Consultant Psychiatrist at the Royal Edinburgh Hospital.
Epilim, was well established and second-line use is not a restriction imposed on, nor observed with, unlicensed products.

\section{Teratogenicity of lithium versus valproate}

The discrepant perception of risk-benefit between lithium and valproate is most starkly highlighted in relation to teratogenicity (see supplementary File 1 for additional references, available at https:// doi.org/10.1192/bjp.2019.137). The Register of Lithium Babies, established in the late 1960s to collect data on the risks of in utero exposure, reported information on 225 babies, 25 (11\%) of whom had birth defects, 18 of which were cardiovascular, including 6 (2.7\%) with Ebstein's anomaly. Although these data suggested a lower, more restricted risk of birth defects than anticipated, they were in reality grossly inflated by powerful reporting bias - voluntary submissions were more likely for affected than unaffected infants. Nonetheless, they were highly influential in imparting to lithium an almost unique perception of teratogenicity among psychotropics. More recent assessment does not clear lithium entirely, suggesting a 1:1500 exposures risk of Ebstein's anomaly, ${ }^{1}$ although with extremely rare events the reliability of such estimates is questionable. Furthermore, available data may not yet have captured any minimising effect of the generally lower therapeutic blood levels now recommended. Contrary to perception, major cardiac malformations attributable to in utero lithium exposure are very rare, while mild anomalies often resolve spontaneously. Importantly, in utero exposure is not associated with other major abnormalities. ${ }^{1}$

The situation with valproate is strikingly different. Despite increased use in young women, its extensive teratogenic risks have been known for many years, including up to a 20 -fold increase in neural tube fusion deficits, especially lumbosacral meningomyelocele, cleft lip/palate, cardiovascular abnormalities, skeletal/limb malformations (including bilateral radial aplasia) and genitourinary defects (including a $2 \%$ risk of hypospadias in males). Overall, structural and organ deficits affect around $10 \%$ of the exposed offspring of women with epilepsy, the risk seemingly dose related, although the highly variable kinetics of valproate in women of childbearing 
age contributes to complexity in establishing a threshold. Even accepting the possibility of lower dose utilisation in psychiatric contexts, this amounts to a more general embryopathic risk than from lithium or other mood stabilisers.

Additionally, an increasing epilepsy literature suggests negative developmental consequences following in utero valproate exposure. $^{2}$ The most consistent findings relate to impairment of global cognitive abilities (average IQ reduction of 8-11 points), an approximately threefold increase in autism spectrum disorder and fivefold increase in autism. An increased risk of attention-deficit hyperactivity disorder is also reported. No critical exposure period is known for these deficits but their nature is compatible with ongoing drug effects throughout gestation. Such developmental disorders, which may affect up to $40 \%$ of infants exposed in utero, have not been attributed to lithium use in pregnancy, although the literature comprises only two small studies.

In interpreting these findings, an important confound is the potentially adverse effects of the underlying illness on pregnancy outcomes. This issue, much discussed in the neurological literature and probably largely accounted for, has been less researched in psychiatric contexts. There is evidence that untreated bipolar disorder is associated with adverse pregnancy outcomes, although the limited data do not highlight a theme of organ dysgenesis. In psychiatric contexts, it would seem erroneous to allow this to distract from clinically important drug effects.

\section{Recent regulatory changes}

As valproate's psychiatric use increased, evidence suggested doctors were failing in their responsibility to inform patients of the risks associated with in utero exposure. In 2014, solid evidence confirming the magnitude of risk and its validity as a drug effect, plus the general failure to communicate this across disciplines, stimulated regulatory action in Europe, including changes to product information, implementation of risk minimisation measures and circulation of an educational direct healthcare professional communication. The response was disappointing, with prescription volumes remaining stable. In 2017, a further European Union-wide review was instigated and on 24 April 2018, the UK regulator, the Medicines and Healthcare products Regulatory Agency, announced widely publicised changes to the conditions for valproate use. Specifically, the drug now carries a contraindication in pregnancy and women of childbearing potential. The only exception is for those participating in the pregnancy prevention programme. Both patient (or their legal guardian) and specialist doctor must sign an 'acknowledgement of risk' form, confirming that the risks have been explained and understood, and committing to specialist review, at least annually. Patients must accept 'effective' contraception. The regulator, sensitive to the consequences of prodromal bipolar relapse on judgement, requires that 'effective contraception' does not rely solely on self-administered methods (for example condoms, contraceptive pill).

The effectiveness of these stringent measures remains to be evaluated but any compulsory prescribing restrictions are open to circumvention and it would be complacent to rely on them alone as sufficient. A more balanced appraisal of valproate's place in psychiatric practice can only come from a change in perception, where risk is realistically set against benefit. Unlike with epilepsy, where a group of patients exists in whom adequate treatment goals cannot be achieved without valproate, no such subgroup of patients with bipolar disorder is known. In psychiatric practice, there are alternatives, including not just lithium but antipsychotics. In patients whose condition is intractable to medication, electroconvulsive therapy can be safe, even in early pregnancy. ${ }^{3}$ This weighs clinical judgement much more towards valproate's risks when the indications in female patients are psychiatric rather than neurological.

\section{Further causes for concern about valproate}

Although pregnancy offers a window into aspects of genotoxicity, additional evidence encourages caution with a drug that may seem benign but has complex, poorly defined pharmacodynamics (see supplementary File 1 for additional references). As a small cationic molecule, lithium's potential to exert multifarious actions is appreciated, if poorly understood. However, while on the surface seemingly relatively well tolerated, valproate also has the potential to disrupt fundamental physiological processes beyond the womb that practice trends suggest are less readily acknowledged. Three examples illustrate the point.

Both drugs can promote weight gain but increases are greater with valproate ${ }^{4}$ and can be associated with dyslipidaemia and insulin resistance, which have not been found as a direct action of lithium. Indeed, lithium increases glucose transportation and glycogen synthesis in insulin-sensitive mammalian muscle, possibly related to potent inhibition of glycogen synthase kinase-3, prompting recommendations for its use in non-insulin dependent diabetes. For a profession in which metabolic dysfunction with antipsychotics is a prominent safety concern, absence of wide discussion of this issue with valproate is striking.

Valproic acid exerts epigenetic actions via potent histone deacetylase inhibition. Gene transcription is regulated by conformational changes in chromatin resulting from the acetylation states of lysine and arginine residues of histone - an 'open' conformation favouring transcription, a 'closed' one being unfavourable. Switching between the two is mediated by reversible deacetylation of histones, effected by histone acetyltransferase and histone deacetylase, a process tightly controlled. Acetylation/deacetylation equilibrium is crucial to brain development, disruption being one candidate for valproate's in utero effects, and its loss has also been implicated in disease states. Histone deacetylase inhibitors are an active field of pharmacological research, including in psychiatry, but the processes remain poorly understood and the consequences of destabilising acetylation homeostasis unknown, especially on the brain, which continues maturation well into the social definition of adulthood. As inhibition of acetylation impairs sperm motility, this may be one mechanism underlying reduced fertility in valproate-exposed male patients. Beyond this, the consequences of valproate's epigenetic actions on fetal development when exposure is paternal are unknown but require exploration.

Reduced male fertility when on valproate also reflects profound neuroendocrine changes that it can mediate, raising dehydroepiandrosterone levels and lowering gonadotropin. In addition, however, the suggestion that valproate may be associated with development of the major endocrine disorder, polycystic ovary syndrome, in female patients with epilepsy, first raised 25 years ago, persists. This has proved difficult to establish owing to variable definitions, exposure durations etc, but meta-analysis of data using various criteria suggests a 1.95 -fold increase in female patients who have epilepsy and are treated with valproate over those on other antiepileptics, ${ }^{5}$ supporting a valproate effect. The risk in bipolar disorders is hard to quantify owing to disconcertingly limited data but prevalences of $10 \%$ have been reported in patients treated with valproate. One consistent finding is that polycystic ovary syndrome changes are more likely to become evident in female patients exposed at a younger age. In view of the profound impact of polycystic ovary syndrome and related endocrine changes on reproductive health, 
this fact, little commented on in the psychiatric literature, must stimulate debate about whether valproate is ever a suitable treatment for bipolar disorder in adolescent and young female patients, regardless of potential teratogenic effects on future pregnancies which, even when planned, may be hard to achieve.

\section{Key unanswered questions}

Valproate is undoubtedly useful in some patients with bipolar affective disorder but their number is likely to be much smaller than current practice suggests. Pragmatic research is needed to address outstanding questions crucial to establishing its safe use in psychiatric patients - does a preferentially responding group exist: if so, what are its characteristics: how long must exposed patients, especially but not exclusively female patients, be off valproate before we can have confidence that its epigenetic and hormonal actions have fully reversed? And what precisely are the neurodevelopmental consequences of its use throughout the lifespan, especially following early exposure?

\section{Conclusions}

Although the 'benefit' side of the therapeutic equation may justify the tendency of international guidelines to present treatment options for acute manic episodes with equivalence, 'risk' considerations do not.

Notwithstanding its own problems, lithium should be prioritised unequivocally as first-line treatment for both acute and maintenance phases of bipolar affective disorders. With the known resistance of patients to changing established medications, starting acute treatment with valproate increases the likelihood of its continuation into maintenance, a role in which it is clearly less efficacious. Routine blood monitoring, so integral to lithium use, should be extended to valproate, to include metabolic and hormone parameters, especially in female patients. Psychiatry must change its perception of valproate, concentrating less on how 'easy' its use appears to be and focusing more on its diverse and poorly understood 'risks'. The teratogenic issue, important in its own right, shines a light on the many other unanswered questions about the place of this drug in modern psychiatric practice and the research still required to address them.

David Cunningham Owens, Professor of Clinical Psychiatry and Honorary Consultant Psychiatrist, Centre for Clinical Brain Sciences, Division of Psychiatry, University of Edinburgh, Royal Edinburgh Hospital, Morningside Park, Edinburgh EH10 5HF, UK. Email: david.owens@ed.ac.uk

First received 20 Aug 2018, final revision 7 May 2019, accepted 15 May 2019

\section{Supplementary material}

Supplementary material is available online at https://doi.org/10.1192/bjp.2019.137.

\section{References}

1 Ornoy A, Weinstein-Fudim L, Ergaz Z. Antidepressants, antipsychotics and mood stabilisers in pregnancy: what do we know and how should we treat pregnant women with depression. Birth Defects Res 2017; 109: 933-56.

2 Meador K, Reynolds MW, Crean S, Fahrbach K, Probst C. Pregnancy outcomes in women with epilepsy: a systematic review and meta-analysis of published pregnancy registries and cohorts. Epilepsy Res 2008; 81: 1-13.

3 Ward HB, Fromson JA, Cooper JJ, De Oliviera G, Almeida M. Recommendations for the use of ECT in pregnancy: literature review and proposed clinical protocol. Arch Womens Ment Health 2018; 21: 715-22.

4 Abosi O, Lopes S, Schmitz S, Fiedorowicz JG. Cardiometabolic effects of psychotropic medications. Horm Mol Biol Clin Investig 2018; 36: 1-15.

5 Hu X, Wang J, Dong W, Fang Q, Hu L, Liu C. A meta-analysis of polycystic ovary syndrome in women taking valproate for epilepsy. Epilepsy Res 2011; 97: 73-82. 